\title{
Eberhard W. Sauer, Hamid Omrani Rekavandi, Tony J. Wilkinson, Jebrael Nokandeh. Persia's Imperial Power in Late Antiquity. The Great Wall of Gorgan and Frontier Landscape of Sasanian Iran
}

Rémy Boucharlat

\section{OpenEdition}

\section{Journals}

Édition électronique

URL : http://journals.openedition.org/abstractairanica/41492

DOI : $10.4000 /$ abstractairanica. 41492

ISSN : 1961-960X

Éditeur :

CNRS (UMR 7528 Mondes iraniens et indiens), Éditions de l'IFRI

Référence électronique

Rémy Boucharlat, « Eberhard W. Sauer, Hamid Omrani Rekavandi, Tony J. Wilkinson, Jebrael Nokandeh. Persia's Imperial Power in Late Antiquity. The Great Wall of Gorgan and Frontier Landscape of Sasanian Iran », Abstracta Iranica [En ligne], Volume 34-35-36 | 2017, document 1, mis en ligne le 15 juillet 2016, consulté le 30 septembre 2020. URL : http://journals.openedition.org/abstractairanica/ 41492 ; DOI : https://doi.org/10.4000/abstractairanica.41492

Ce document a été généré automatiquement le 30 septembre 2020

Tous droits réservés 


\title{
Eberhard W. Sauer, Hamid Omrani Rekavandi, Tony J. Wilkinson, Jebrael Nokandeh. Persia's Imperial Power in Late Antiquity. The Great Wall of Gorgan and Frontier Landscape of Sasanian Iran
}

\author{
Rémy Boucharlat
}

\section{RÉFÉRENCE}

Eberhard W. Sauer, Hamid Omrani Rekavandi, Tony J. Wilkinson, Jebrael Nokandeh. Persia's Imperial Power in Late Antiquity. The Great Wall of Gorgan and Frontier Landscape of Sasanian Iran. British Institute of Persian Studies, Archaeological Monographs Series II, Oxbow Books, Oxford and Oakville, 2013, XVI-712 p., 448 fig., nombreux tableaux, $18 \mathrm{Pl}$. coul., bibliographie, résumé en persan $5 \mathrm{p}$.

Sous ce titre, les AA. rendent compte du programme de fouille irano-britannique conduit entre 2005 et 2009, publié à peine quatre ans après dans un livre de grande qualité par son contenu, soutenu par une édition magnifique. Les travaux ont porté à la fois sur le mur de Gorgān, une barrière ouest-est depuis la mer Caspienne jusqu'aux forêts dans les montagnes sur près de $200 \mathrm{~km}$, et sur le mur de Tammī̌se, perpendiculaire au premier, sans doute à partir du même point d'origine, aujourd'hui dans la mer, et qui du nord au sud ferme l'étroite bande de terre entre mer et montagnes de l'Alborz. Parmi les acquis très importants de ces travaux, figure la relation étroite entre les deux murs - le second a souvent été considéré comme plus récent - et (enfin!) des séries de datations concordantes, entre le milieu du $\mathrm{V}^{\mathrm{e}}$ et le 
début du VIe s. de l'ère chrétienne. Par l'analyse de la documentation historique, les AA. proposent d'en attribuer la construction à Yazdigird II (438-457) ou Pērōz (457-484), qui tous deux sont longuement intervenus dans la région, avant que ce dernier n'y trouve la mort contre les Hephtalites. Voilà donc une question enfin réglée.

2 Un autre point très important est celui de la fonction de ces murs, celui de Gorgān principalement. Ce sont évidemment des barrières, car les murs sont puissamment renforcés par des tours, des fortins en arrière et, de place en place, des forts, des bases militaires comme les qualifient les AA., dans lesquels pouvaient loger une garnison et plus de soldats en cas de nécessité. Des villes ont été construites, comme celle du site actuel de Dašt-e Qal'e. Le long du mur de Gorgān un canal dérivait l'eau de l'Atrak, formant un fossé défensif, mais, de là, partaient des canaux plus petits destinés à l'irrigation des terres en deçà et au-delà du mur. Comme le montre bien ce livre, le mur de Gorgān n'est pas une barrière passive, mais il est aussi ou d'abord un énorme projet de mise en valeur d'une région frontière de part et d'autre du mur.

3 Dans les quatre sections, la première, est une longue et nécessaire introduction à l'histoire de la recherche et aux hypothèses de chronologie proposée depuis 150 ans. La seconde section, est la plus fournie. D'abord une étude de l'environnement dirigée par feu Tony Wilkinson, géoarchéologue, grand spécialiste du Proche et Moyen-Orient. L'étude fine du peuplement antérieur et contemporain, celle des canaux et dérivations sont évidemment très importants pour mesurer l'investissement décidé par la puissance royale. Puis sont présentés les rapports sur les prospections géophysiques, très fructueuses, les sondages et fouilles de trois fours à briques - il y en avait plusieurs milliers tout le long du mur, l'architecture du mur lui-même, les fortins et tours de guet, les forts, les bases militaires en arrière. Ces dernières sont bâties sur un modèle régulier, tous pourvus d'une citadelle. Enfin, la ville neuve de Dašt-e Qal'e qui auraient $\mathrm{pu}$ abriter des dizaines de milliers d'habitants, mais il n'est pas sûr qu'elle ait été densément occupée dans la réalité, pas plus que ne l'ont été d'autres fondations royales des Sassanides.

4 Au-delà du mur, un établissement de l'âge du Bronze a été sondé, confirmant l'occupation importante de la région à cette époque. L'âge du Fer et peut-être l'époque achéménide y sont aussi représentés.

Dans la troisième section, on retient surtout l'étude la faune, intéressante pour la connaissance du régime alimentaire de ces époques historiques pour lesquelles ce type d'étude est encore trop rare. L'étude de la céramique est très détaillée, bien menée, et sera très utile pour les travaux futurs dans la région, située entre le Plateau iranien et l'Asie centrale méridionale.

6 La dernière section replace l'histoire et la raison d'être de ces murs dans la conception des grandes murailles anciennes, des murs romains d'Hadrien et Antonin à la frontière de l'Angleterre et de l'Ecosse à la grande muraille de Chine. Pour répondre à une interrogation des auteurs sur la forme de l'empire sassanide, confédération lâche ou empire centralisé, il me semble qu'une entreprise monumentale comme celle du mur de Gorgān illustre clairement la seconde hypothèse : souci de protection d'une frontière et tout autant celui de la mise en valeur d'une région éloignée des centres politiques et économiques de Mésopotamie et d'Iran méridional. 


\section{AUTEURS}

RÉMY BOUCHARLAT

CNRS, Lyon 